\title{
Evaluation of Congenital Heart Disease by Three-dimensional Magnetic Resonance Imaging
}

\author{
Michael W. Vannier, Fernando R. Gutierrez, Charles E. Canter, Charles F. Hildebolt, Thomas K. Pilgram, \\ Robert C. McKnight, John C. Laschinger, Jeffrey J. Brown, Scott A. Mirowitz, Bradley D. Raisher, \\ Daniel C. Sekarski, and Roberta L. Yoffie
}

\begin{abstract}
This study was undertaken to compare electrocardiographically gated magnetic resonance imaging (MRI) to established imaging modalities in the detection of complex intra- and extracardiac morphologic defects. Twenty-three patients with congenital cardiac abnormalities were imaged by four methods: cardiac catheterization, echocardiography, two-dimensional (2D) transaxial MRI, and three-dimensional (3D) MRI surface reconstruction. Observers with experience in congenital cardiac disease diagnosis (two for echo, one for catheterization, two for 2D MR, and three for 3D MR) evaluated the images in a blinded fashion, and the results were analyzed with receiver operating characteristic (ROC) analysis. Overall, cardiac catheterization had the best diagnostic performance. The diagnostic value of routine 2D cardiac MR images and 3D MR reconstruction images were similar to that of echocardiography. All of the modalities performed poorly in the diagnosis of extracardiac defects and atrial septal defects.
\end{abstract}

Copyright $₫ 1991$ by W.B. Saunders Company

KEY WORDS: magnetic resonance, comparative studies, magnetic resonance, in infants and children, heart, congenital heart disease.

@TANDARD CONGENITAL cardiovascular diagnostic imaging modalities such as plain film radiography, echocardiography, angiography, scintigraphy, and computed tomography have been supplemented by the recent development of electrocardiogram [ECG]-triggered magnetic resonance imaging (MRI). ${ }^{1} \mathrm{MRI}$ is very effective at delimiting the natural boundary that exist between flowing blood and cardiovascular structures ${ }^{2-10}$ because flowing blood fails to produce an MR signal. As a result, high resolution two-dimensional (2D) images of cardiac and vascular structures may be obtained noninvasively, without exposure to ionizing radiation and without the need for intravascular contrast.

These imaging techniques have been used successfully to diagnose a wide variety of congenital cardiovascular disorders and morphologic malformations, and to assess cardiac function. However, the planar format of the standard modalities is not ideal for fully comprehending the underlying three-dimensional (3D) cardiac morphology. This is particularly true in patients with complex congenital cardiovascular malformations, in which surgical planning is based on the successful transfer of information from the radiologist to the surgeon and on the surgeon's ability to mentally reconstruct a single complex anatomy from multiple 2D images.

Specialized computer software methods, originally developed for the preoperative evaluation of complex craniofacial deformities ${ }^{11,12}$ have been adapted to allow display of 3D surface reconstruction images of the heart from 2D MR scan data. ${ }^{13-17}$ 3D MR images have demonstrated their potential usefulness in transferring information from the radiologist to the surgeon. ${ }^{18-20}$ The objective of this study was to examine the diagnostic value of 3D MRI by comparing it with 2D MRI, echocardiography, and angiography.

\section{MATERIALS AND METHODS}

\section{Sample}

Twenty-three patients with congenital heart disease were included in this analysis (Table 1). Twenty-two underwent cardiac catheterization, 21 had echocardiograms, 22 had MR scans, and 12 underwent surgery soon after imaging. The patients varied in age from 2 days to 22 years, with 6 patients below the age of 1 year, 14 between 1 and 6 years of age, 2 between 7 and 12 years, and 2 older than 12 years. One 4-year-old individual had a full set of images both before and after surgery and was used twice, so the total sample is 24 hearts from 23 patients.

From the Mallinckrodt Institute of Radiology, Washington University School of Medicine, the Division of Pediatric Cardiology, Department of Pediatrics, and the Division of Cardiothoracic Surgery, Department of Surgery, Washington University School of Medicine, St Louis, MO.

Supported in part by Siemens Medical Systems, Inc, Iselin, NJ.

Address reprint requests to Michael $W$. Vannier, $M D$, Mallinckrodt Institute of Radiology, Washington University School of Medicine, 510 S Kingshighway Blvd, St Louis, MO 63110.

Copyright $\odot 1991$ by W.B. Saunders Company

0897-1889/91/0403-0004\$03.00/0 
Table 1. Cardiac Characteristics of the Sample

\begin{tabular}{lrrr}
\hline & \multicolumn{2}{c}{ Diagnosis } & \\
\cline { 2 - 3 } \multicolumn{1}{c}{ Anatomical Region } & Normal & Abnormal & Total \\
\hline Heart & & & \\
$\quad$ Atrium & 19 & 5 & 24 \\
$\quad$ Left & 17 & 6 & $23^{*}$ \\
$\quad$ Right & & & \\
Ventricle & 18 & 6 & 24 \\
$\quad$ Left & 3 & 20 & $23^{*}$ \\
$\quad$ Right & 10 & 14 & 24 \\
Atrial septal defect & 10 & 13 & 23 \\
Ventricular septal defect & & & \\
Ventricular outflow tract & 18 & 5 & $23^{*}$ \\
$\quad$ Left & 4 & 20 & 24 \\
$\quad$ Right & & & \\
Aorta and Pulmonary Arteries & 10 & 14 & 24 \\
Ascending aorta & 7 & 17 & 24 \\
$\quad$ Aortic root & 11 & 13 & 24 \\
Main pulmonary artery & 14 & 10 & 24 \\
Left pulmonary artery & 17 & 7 & 24 \\
$\quad$ Right pulmonary artery & &
\end{tabular}

*Four patients whose combined information did not permit truth determination for just one anatomical region were included in the study.

\section{Cardiac Catheterization}

Procedures were performed on a Siemens biplane Angioskop with Koordinat angiographic table (Siemens Medical Systems Inc, Iselin, NJ) with all controls assembled in a mobile console. This system was connected to a Hewlett Packard (Andover, MA) 8890B monitoring and end analysis system. A Gigantos Optimatic x-ray generator (Siemens) (fully automatic 12 pulse unit) produced $1000 \mathrm{~mA}$ at $100 \mathrm{kv}$. Cine exposure was obtained with biplane 35MM Arritechno (Munich, Germany) cine cameras at up to 60 frames/sec. Cinerex (Richfield Park, NJ) PFA Agfa-Gevaert film was utilized and processed on a Jamieson (Dallas, TX) Processor (Model 54).

\section{Echocardiography}

During our studies we used an Aloka (Tokyo, Japan) 880 real time echocardiography system for a few studies, but did the bulk of our research with an Aloka 860. Transducer selection varied between a $5 \mathrm{mHz}$ and $3.5 \mathrm{mHz}$ transducer, with the bulk of the studies being performed with the $5 \mathrm{mHz}$ transducer. The test were performed trying to concentrate on minimal depth ranges for higher pulse repetition frequencies (PRF). All studies were done with PRF of $6 \mathrm{kHz}$ or 8 $\mathrm{kHz}$. The depth ranges varied from 6 to 12 centimeters of depth depending upon the patient's size. B-mode scanning angle was kept at $90^{\circ}$ with colorflow angle at $46^{\circ}$. The focal distance varied between 40 and $75 \mathrm{~mm}$, again depending upon patient size. One hundred twenty-eight scanning lines of data were maintained during straight B-mode imaging but were reduced to 64 lines per frame when in the color-mode. We strove to keep the frame rate at a maximum of 30 frames per second (FPS) but pending upon the PRF and depth the frame rate sometimes dropped to a minimum of 15 FPS.
Contour edge enhancement was not used. All information was displayed on a Sony (Sony Corp, New York, NY) 13-inch trinitron color TV monitor using a NTSC (National Television System Committee) (3.58 $\mathrm{MHz}$ ) system. All video recording was performed on standard $1 / 2$ inch VHS 120 tape with a Panasonic (Panasonic Corp, Seacaucus, NJ) 6200VHS recorder.

\section{MRI}

Contiguous ECG-triggered 2D MR images were recorded from the apex of the heart to the aortic arch at several equispaced times in the cardiac cycle for each slice. ${ }^{18}$ These images were obtained using spin-echo sequences at 3 to 5 equi-spaced echo-delay time values on a 0.5 or 1.5 Telsa Siemens Magnetom MR scanner. 3D reconstruction requires that slices be contiguous and be obtained at the same pulse-repetition interval. The scans were obtained with 5 or $10 \mathrm{~mm}$ slice thicknesses and 5 or $10 \mathrm{~mm}$ interslice gaps respectively. A second set of slices was then obtained to fill the $100 \%$ gap intervals resulting in an interleaved stack of image sections with no interslice gap. The acquisition time for a single cardiac study in a child with congenital heart disease whose heart rate averaged 100 beats per minute was 1 hour total. Twelve to 36 contiguous transaxial MR slice images were obtained through the heart in these patients (Fig 1A).

\section{D MRI Processing Techniques}

A sequence of ECG gated contiguous transaxial MR slices of the thorax obtained at the same point in the cardiac cycle were used for $3 \mathrm{D}$ reconstruction processing. This set of 15 images is sufficient to perform a high-resolution 3D surface reconstruction. The same set of MR thoracic transaxial slices at three to seven different times within the cardiac cycle were originally obtained but typically only one set per patient at a single point in the cardiac cycle was analyzed.

Manual segmentation of individual 2D MR images was employed as a preprocessing step before surface reconstruction. This was supplemented with software originally developed for echocardiography and digital subtraction angiography and with specialized software originally developed for processing high-resolution computed tomography scan data (Siemens Evaluscope DR and Somatom DR). The software was adapted to permit rapid isolation of the heart and great vessels in axial cardiac MR scans of the thorax.

Using a set of image editing functions, including selective erasure and region filling, the heart and great vessels were isolated by an anatomically knowledgeable radiologic technologist operator (R. Yoffie). ${ }^{13-15,18,21}$ Each slice was viewed by the operator who selected the appropriate region of interest within each slice. The acceptability of edited MR slice images for 3D surface reconstruction was determined by radiologists (F. Gutierrez and $M$. Vannier), and the final 3D images were checked for correctness by a cardiac radiologist (F. Gutierrez).

For this study, the views obtained in each case included cranio-caudal, caudo-cranial, frontal, rear, lateral (right and left) and four chamber images in the majority of cases (Fig $1 B$ ). The number of 3D views for each case varied from four to eight with six views available for most cases. 

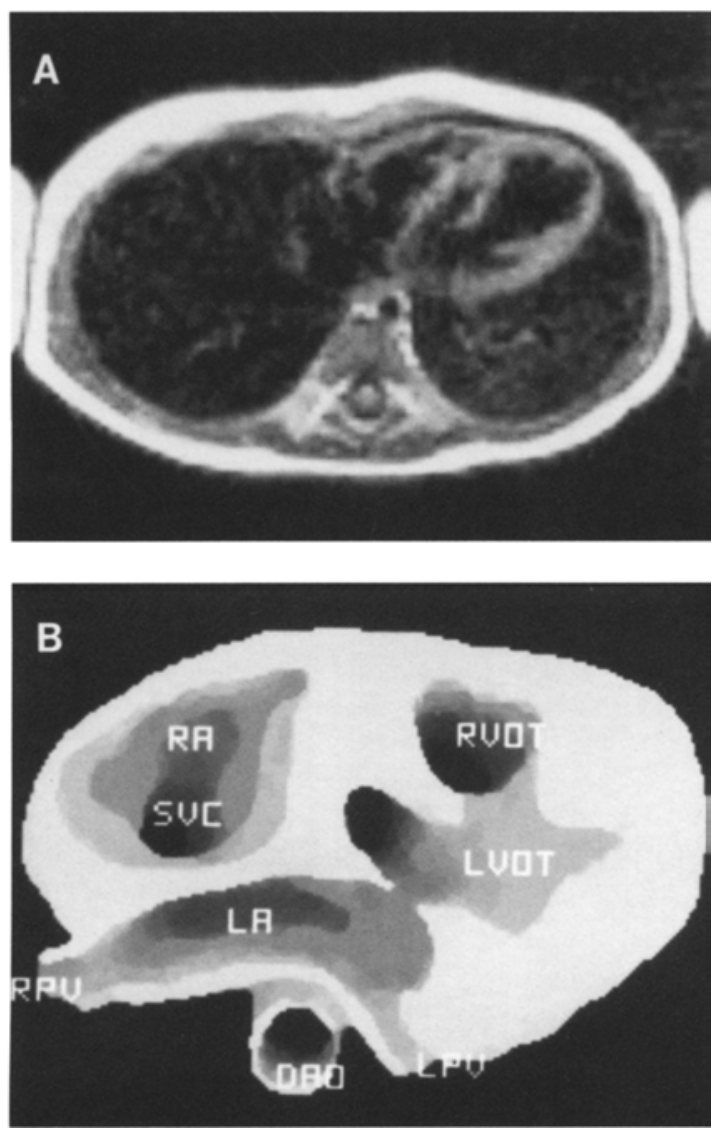

Fig 1. (A) This 7-month-old female has a large ventricular septal defect with right ventricular hypertrophy and enlargement of the main pulmonary artery proximally. This is a large atrio-ventricular canal-type ventricular septal defect. In a representative transaxial slice, the right ventricle is hypoplastic with a thickened wall. This cardiac malformation is essentially a single ventricle. (B) There is communication between the right and left ventricular outflow tracts in this caudalcranial view. Drainage of the superior vena cava into the right atrium can be observed. The mitral valve can be seen between the left atrium and the adjacent left ventricular outflow tract and aortic route.

\section{Truth}

Truth was established by means of consensus of an experienced cardiac radiologist (F. Gutierrez) and pediatric cardiologist (C. Canter), who had access to images from all modalities and to all clinical records, including surgical reports (Table 1 ). The individuals who participated in the establishment of truth did not take part in the rating study.

\section{Observers}

The MR slice examinations and 3D reconstruction images were reviewed by a radiologist (J. Brown) with specialty training in magnetic resonance imaging as well as a senior resident (S. Mirowitz) in diagnostic radiology, experienced in MR diagnosis of congenital heart disease. In addition, 3D MRI examinations were reviewed by a senior fellow in cardiothoracic surgery (J. Laschinger), experienced in MR imaging of congenital heart disease. The echocardiographic examinations were reviewed from video tape by an experienced pediatric cardiology fellow (B. Raisher) and the Technologist Director of the Pediatric Echocardiography Laboratory (D. Sekarski). Cardiac catheterization examinations were read by a cardiac radiologist (R. McKnight).

Patient names, ages, examination dates, and all other individual information was masked on the diagnostic images. All studies were identified with a three-digit number only. Each observer rated 28 cardiac abnormalities on a 5 -point scale ranging from 1 (definitely or almost definitely abnormal) to 5 (definitely or almost definitely normal). ${ }^{22}$ Images were rated only once by each observer.

\section{Analysis}

Data were pooled from all observers; that is, each rating by each observer was treated as if it were a separate observation. ${ }^{18}$ This was done because of the small sample sizes for most defects and the degenerate nature of the ratings of some defects. Receiver operating characteristics (ROC) curves were produced and areas under the curves (A calculated with the ROCFIT program. ${ }^{22,23}$

As a supplement to the ROC analysis, areas under the curves for the modalities were ranked from 1 to 4 for each defect, with 1 being the $A_{z}$ with the largest area and 4 being the $A_{z}$ with the smallest area. The rankings for all defects were then combined as a summary measure of performance across all regions.

\section{RESULTS}

The results of 13 ratings were selected as being representative of the 28 defects (see Tables 2 and 3). Diagnostic performance was generally poor for all modalities. For 4 of 13 regions the greatest $A_{z}$ was less than 0.70 , and for 11 of 13 it was less than 0.80 . Considering that 0.50 would be expected by chance, a large portion of the results indicate only a limited improvement over random diagnosis. The overall pattern is that all of the imaging techniques have high value in the diagnosis of ventricular septal defects but have limited value with regard to diagnoses of defects of the atrial septum, outflow tracts, and extracardiac structures.

It was impossible to fit ROC curves in 12 of 13 cases for cardiac catheterization, due to degenerate data. The degeneracy resulted from a high level of confidence on the part of the single observer. Most of the responses were definite, which caused the curves to be vertical or horizontal at some FPF. In effect, observer confidence transformed a rating experiment into a twoalternative-forced-response experiment. How- 
Table 2. Areas Under the Fitted ROC Curve Heart

\begin{tabular}{lcc}
\hline & Atrium & Ventricle \\
\hline Left & $*$ & \\
Cath & $*$ & $*$ \\
Echo & 0.6431 & 0.6938 \\
MR slice & 0.6807 & 0.7044 \\
3D MR & 0.7696 & 0.6735 \\
Right & & \\
Cath & $*$ & $*$ \\
Echo & 0.6496 & $*$ \\
MR slice & 0.8123 & $*$ \\
3D MR & 0.7809 & 0.6497 \\
Septal defect & & \\
Cath & $*$ & $*$ \\
Echo & 0.7167 & 0.8488 \\
MR slice & 0.5344 & 0.7848 \\
3D MR & 0.6595 & 0.8643 \\
Left outflow tract & & \\
Cath & & 0.7057 \\
Echo & & 0.7699 \\
MR slice & & 0.6411 \\
3D MR & & 0.5518 \\
Right outflow tract & & \\
Cath & & $*$ \\
Echo & & 0.6647 \\
MR slice & & 0.5388 \\
3D MR & & 0.5720 \\
\hline
\end{tabular}

*Degenerate data.

ever, cardiac catheterization consistently provided the greatest proportion of correct answers.

\section{DISCUSSION}

The two most evident patterns in our study are, first, the generally low values of $A_{z}$ for all modalities for most of the diagnostic problems, and second, the relative equivalence of all modalities except cardiac catheterization. To summarize the results in a simple fashion, modalities were ranked by their value for $\mathrm{A}_{z}$ for each diagnostic test, and a frequency distribution calculated for the rankings (Fig 2). Cardiac catheterization is not included because the results were usually degenerate. Where it is left out the ranks began at 2 . For the 13 diagnostic tests selected, Echo, 3D MR, and MR slice were about equal in diagnostic value.

\section{LIMITATIONS}

This study has several limitations. First, it is a nonrandomized retrospective study, and second, the patients were not examined under strict uniform protocols. For example, the slice thickness and number of reconstructed $3 \mathrm{D}$ views varied from case to case. The examinations were performed in a nonrandom order, and the sample size is small with a significant overrepresentation of abnormals. The patients who were included were generally much younger and had more complex lesions evaluated than series examined with MR reported elsewhere..$^{10,24}$ The quality of the examinations is nonuniform and reflects the experience gained with the modalities over the term of the study.

The physicians responsible for truth reported that, subjectively, cardiac catheterization was the most heavily weighted factor used for establishing truth. The apparent superiority of cardiac catheterization to other modalities may, therefore, simply indicate that there was a high degree of agreement between the observer who interpreted cardiac cath studies blindly and those who used cardiac cath to establish truth.

Surgical reports were used to partially control for this problem; yet in many instances, no information was available concerning possible cardiac defects beyond those for which the operation was performed (for instance, if the operation was performed to correct a ventricular septal defect, little if any information was collected at the time of surgery on extracardiac

Table 3. Areas Under the Fitted ROC Curve Aorta and Pulmonary Arteries

\begin{tabular}{lc} 
Ascending aorta & $*$ \\
Cath & 0.6820 \\
Echo & 0.5322 \\
MR slice & 0.5452 \\
3D MR & \\
Aortic root & $*$ \\
Cath & 0.7615 \\
Echo & 0.6083 \\
MR slice & 0.5361 \\
3D MR & $*$ \\
Main pulmonary artery & \\
Cath & 0.4018 \\
Echo & 0.7524 \\
MR slice & 0.6030 \\
3D MR & \\
Left pulmonary artery & $*$ \\
Cath & 0.5584 \\
Echo & 0.6879 \\
MR slice & 0.6004 \\
3D MR & \\
Right pulmonary artery & 0.4184 \\
Cath & 0.5839 \\
Echo & \\
MR slice & \\
3D MR & \\
\hline
\end{tabular}

*Degenerate data. 


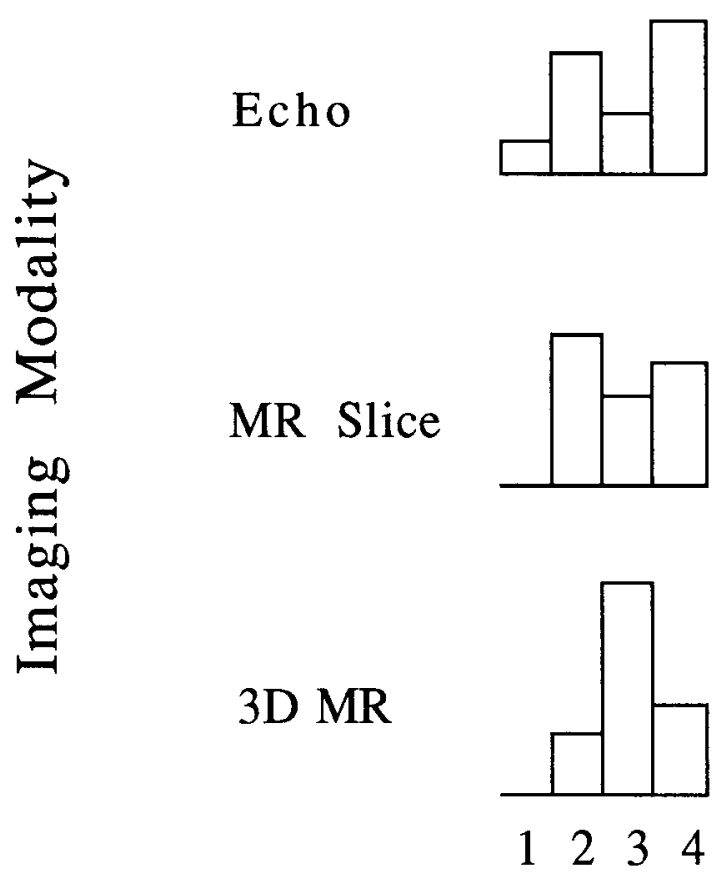

Rank

Fig 2. The values of the areas under the fitted ROC curves $\left(A_{2}\right)$ were ranked for echocardiography, 2D transaxial MR, and 3D MR surface reconstruction, and a frequency distribution calculated for the rankings. Cardiac catheterization was not included because $A_{2}$ could not be calculated in most cases, but where it is left out the other rankings begin at 2.

defects.) A possible solution to this problem would be to base truth on cardiac prosection of fatalities. No such cases were available for our study.

\section{CONCLUSION}

With regard to the ability of conventional diagnostic imaging methods to diagnose atrial septal defects, all modalities performed poorly and were approximately equal in diagnostic value. For diagnosis of ventricular septal defects, all modalities provided satisfactory results, with cardiac catheterization being of the highest diagnostic value.

The most important conclusion that can be drawn from this study is that the use of images in the diagnosis of congenital heart deformities is a complex exercise. Unlike many radiographic skeletal diagnoses such as fractures,${ }^{25}$ where the image is the deciding factor, the diagnosis of cardiac abnormalities is strongly influenced by information in addition to that provided by the images. The exclusion of information about medical history is artificial in any study, but it may be most artificial in studies of the heart.

An additional complexity is the nature of the problem. A congenitally deformed heart is not a normal organ that has been damaged, as is, for instance, a broken bone. It is an organ that is abnormal and behaves as such. Because the functional abnormality lies in the pattern of blood flow, images of flow are likely to give the best diagnosis. Though cardiac morphology is the source of the dysfunction, it is not in itself the problem, and images of morphology are unlikely to ever be as diagnostically useful as images of flow.

3D MRI is good at imaging morphology, and comparable in its diagnostic utility to other techniques that do this. Considering the noninvasiveness of MRI, and the ability of 3D MRI to clearly illustrate complex cardiac anatomy, 3D MRI may in many instances be a viable supplement or alternative to traditional cardiac imaging methods. Certainly, further study of its capabilities is warranted.

\section{ACKNOWLEDGMENT}

Manuscript preparation by M. Akin is gratefully appreciated.

\section{REFERENCES}

1. AMA Council on Scientific Affairs: Magnetic resonance imaging of the cardiovascular system, present state of the art and future potential, JAMA 259:259-263, 1988

2. Higgins $\mathrm{CB}$, Byrd BF, Farmer D, et al: MRI in patients with congenital heart disease. Circulation 70:851-860, 1984

3. Fletcher BD, Jacobstein MD, Nelson AD, et al: Gated MRI of congenital cardiac malformations. Radiology 150: 137-140, 1984

4. Didier D, Higgins CB, Fisher MR, et al: Gated MRI in congenital heart disease: Experience in 72 patients. Radiology $158: 227-235,1986$
5. Herfkens RJ, Higgins CB, Hricak H, et al: MRI of the cardiovascular system: Normal and pathologic findings. Radiology 147:747-759, 1983

6. Lanzer P, Botvinick EH, Schuller NB, et al: Cardiac imaging using gated magnetic resonance. Radiology 150:121127,1984

7. Higgins CB: Overview of MR of the heart-1986. Am J Roentgenol 146:907-918, 1986

8. Kersting-Sommerhoff BA, Sechtem UP, Higgins CB: Evaluation of pulmonary blood supply by nuclear magnetic resonance in patients with pulmonary atresia. J Am Coll Cardiol (1):166-171, 1988 
9. Sechtem U, Pflugfelder P, Cassidy MC, et al: Ventricular septal defect: Visualization of shunt flow and determination of shunt size by cine magnetic resonance imaging. Am J Roentgenol 149:689-692, 1987

10. Kerstig-Somerhof BA, Diethelm L, Teitel DF, et al: Magnetic resonance imaging of congenital heart disease: Sensitivity and specificity using receiver operating characteristic curve analysis. Amer Heart J 118:155-161, 1989

11. Vannier MW, Marsh JL, Warren JO: Three dimensional CT reconstruction images for craniofacial surgical planning and evaluation, Radiology 150:179-184, 1984

12. Vannier MW, Marsh JL, Warren JO: Three dimensional computer graphics for craniofacial surgical planning and evaluation. Comput Graphics 17:263-273, 1983

13. Laschinger JL, Vannier MW, Gronemeyer S, et al: Noninvasive three-dimensional reconstruction of the heart and great vessels by ECG-gated magnetic resonance imaging: A new diagnostic modality. Annals of Thoracic Surgery 45:505-514, 1988

14. Laschinger JC, Vannier MW, Gronemeyer S, et al: Three dimensional reconstruction of the heart using EKGtriggered magnetic resonance images. J Med Imag 1:228235,1987

15. Laschinger JC, Vannier MW, Gutierrez FR: Preoperative three-dimensional reconstruction of the heart and great vessels in patients with congenital heart disease. $J$ Cardiothoracic Cardiovasc Surg 96:464-473, 1988

16. Axel L, Herman GT, Udupa JK, et al: Three dimensional display of NMR cardiovascular images. J Comput Assist Tomog 7:172-174, 1983
17. Weinberg PM, Trivedi S, Clark BJ, et al: Threedimensional reconstruction of complex congenital heart defects from magnetic resonance imaging. Circulation 74:II380, 1986 (suppl II)

18. Vannier MW, Gutierrez FR, Laschinger JC, et al: Three-dimensional magnetic resonance imaging of congenital heart disease. Radiographics 8:857-871, 1988

19. Mirowitz SA, Gutierrez FR, Canter CE, et al: Tetralogy of Fallot: MR findings Radiology 171:207-212, 1989

20. Canter CE, Gutierrez FR, Mirowitz SA: Evaluation of pulmonary arterial morphology in cyanotic congenital heart disease by magnetic resonance imaging. Am Heart J 118:347-354, 1989

21. Yoffie RL, Vannier MW, Gutierrez FR, et al: Threedimensional magnetic resonance imaging of the heart. Radiologic Technology 60:305-309, 1989

22. Swets JA, Pickett RM: Evaluation of Diagnostic Systems. New York, NY, Academic Press, 1982

23. Metz CE, Wang DL, Kronman HB: A new approach for listing the significance of differences between ROC curves measured from correlated data, in Deconink $F$ (ed): Information processing in medical imaging. The Hague, Nijhoff, 432-445, 1984

24. Lowell DG, Turner DA, Smith SM: The detection of atrial and ventricular septal defects with electrocardiographically synchronized magnetic resonance imaging. Circulation 73:89-94, 1986

25. Vannier MW, Hildebolt CF, Gilula LA, et al: Calcaneal and pelvic fractures: Diagnostic evaluation by threedimensional computed tomography scans. J Digit Imag 4:143-152, 1991 